\title{
Transient Hyperthyroidism of Hyperemesis Gravidarum: A Sheep in Wolf's Clothing
}

\author{
CPT Timotby J. Caffrey, MC, USA
}

Background: Transient hyperthyroidism of hyperemesis gravidarum (THHG) is a self-limiting hyperthyroidism occurring in the context of hyperemesis gravidarum.

Methods: A literature search of MEDLINE was undertaken, and a case report of a woman with THHG in pregnancy is described.

Results and Conclusions: Because thyroid function tests cannot distinguish Graves disease from THHG, the diagnosis of THHG rests largely on the concurrent development of hyperemesis and hyperthyroidism and the absence of signs and symptoms of hyperthyroidism before and during pregnancy. THHG might be responsible for $\mathbf{4 0 \%}$ to $70 \%$ of thyroid function abnormalities in pregnancy. Both the thyroid function abnormalities and hyperemesis are related to elevated levels of human chorionic gonadotropin. THHG resolves by 18 weeks of pregnancy without sequelae. No treatment is required. Diagnosis of THHG by the primary care provider can prevent unnecessary treatment or referral for specialty care. (J Am Board Fam Pract 2000;13:35-8.)

Transient hyperthyroidism of hyperemesis gravidarum (THHG) is a self-limiting hyperthyroidism occurring in the context of hyperemesis gravidarum. Thyroid function tests in THHG can be indistinguishable from those seen in pathologic causes of hyperthyroidism such as Graves disease. Including THHG in the differential diagnosis of hyperthyroidism in pregnancy can prevent unnecessary treatment or referrals.

\section{Methods}

A case report of a woman with THHG is described, and a literature search of MEDLINE was undertaken. THHG has been only tangentially described in the family practice literature. ${ }^{1}$ The following case report describes a classic case of THHG. A discussion of the clinical findings and diagnosis of THHG ensues. The incidence, mechanism, natural history, and treatment of this condition are also described.

\section{Case Report}

A 32-year-old woman, gravida 4, para 2, came to the emergency department with a chief complaint

Submitted, revised, 27 January, 1999.

From the US Army Health Clinic - Vicenza, Italy. Address reprint requests to CPT Timothy J. Caffrey, MD, US Army Medical Corps, US Army Health Clinic - Vicenza, CMR 427, Box 44, APO, AE 09630. of 2 weeks of emesis, 5 to 6 episodes per day. She felt lightheaded and noted a decrease in both volume and frequency of urination. She had a normal menstrual period 10 weeks before her visit and a positive pregnancy test 3 weeks earlier. She denied nervousness, diaphoresis, heat intolerance, hyperactivity, tremor, weakness, and diarrhea both before and during this pregnancy.

Her obstetric history was notable for two fullterm, vaginal deliveries and a first-trimester miscarriage. She had been hospitalized for hyperemesis early in the course of her first full-term pregnancy. She had no personal or family history of thyroid disease or autoimmune disorders.

Her supine pulse was $98 \mathrm{~mm} \mathrm{Hg}$ that increased to $114 \mathrm{~mm} \mathrm{Hg}$ when standing. Her weight was 10 pounds less than her prepregnancy weight of 125 pounds. She had dry oral mucosa and a slightly enlarged uterus. She had no palpable thyroid abnormalities and no proptosis.

Her urine pregnancy test was positive. Results of a urinalysis were a specific gravity of 1.030 and increased ketones. Serum chemistry was notable for a slightly decreased potassium level of $3.4 \mathrm{mEq} / \mathrm{L}$ (lower limit of normal $3.5 \mathrm{mEq} / \mathrm{L}$ ) and decreased bicarbonate of $21 \mathrm{mEq} / \mathrm{L}$ (lower limit of normal 24 $\mathrm{mEq} / \mathrm{L})$.

A pelvic sonogram confirmed a single, intrauterine pregnancy of 10 weeks and 3 days' gestation. 
Table 1. Patterns of Thyroid Function Studies and Thyroid Autoantibodies in Normal Pregnancy, Transient Hyperthyroidism of Hyperemesis Gravidarum (THHG), and Graves Disease.

\begin{tabular}{|c|c|c|c|}
\hline Thyroid Function Tests & Normal Pregnancy & THHG & Graves Disease \\
\hline Thyroid-stimulating hormone & Normal & Low or normal & Low \\
\hline Thyroid-binding globulin & High & High & Normal or high \\
\hline Total thyroxine & High & High & High \\
\hline Free thyroxine & Normal & High or normal & High \\
\hline Total triiodothyronine & High & High & High \\
\hline Free triiodothyronine & Normal & High or normal & High \\
\hline \multicolumn{4}{|l|}{ Thyroid autoantibodies } \\
\hline Antithyroid peroxidase & Negative & Negative & Positive \\
\hline $\begin{array}{l}\text { Antithyroid-stimulating } \\
\text { hormone receptor }\end{array}$ & Negative & Negative & Positive \\
\hline
\end{tabular}

Her condition was diagnosed as hyperemesis gravidarum and treated with volume replacement and antiemetics. Thyroid function studies were done to rule out hyperemesis secondary to hyperthyroidism. Her initial thyroid-stimulating hormone (TSH) level was $<0.06 \mu \mathrm{U} / \mathrm{mL}$ (lower limit of normal, $0.32 \mu \mathrm{U} / \mathrm{mL})$ and free thyroxine $\left(\mathrm{T}_{4}\right)$ level was $4.16 \mathrm{ng} / \mathrm{dL}$ (upper limit of normal, 2.5 $\mathrm{ng} / \mathrm{dL}$ ). Because the thyroid function studies were consistent with hyperthyroidism, she was referred for treatment of hyperthyroidism in pregnancy.

Since neither her prepregnancy history nor initial findings showed signs or symptoms of hyperthyroidism, an alternative diagnosis of THHG was considered. Further laboratory data showed an elevated free triiodothyronine $\left(T_{3}\right)$ level of 674 $\mathrm{pg} / \mathrm{dL}$ (upper limit of normal, $420 \mathrm{pg} / \mathrm{dL}$ ). An antiTSH receptor antibody titer was negative.

Given the absence of prepregnancy hyperthyroidism, the pattern of elevated thyroid function tests occurring in the context of hyperemesis, the lack of stigmata of hyperthyroidism, and absence of anti-TSH receptor antibodies, a diagnosis of THHG was made. Supportive treatment for hyperemesis was continued. Her symptoms had resolved by 15 weeks' estimated gestational age. $\mathrm{TSH}$, free $\mathrm{T}_{3}$ and free $\mathrm{T}_{4}$ levels returned to normal shortly thereafter. She gave birth to a healthy infant at term.

\section{Discussion}

\section{Evaluation and Diagnosts}

Hyperemesis gravidarum is a condition of persistent and excessive vomiting developing in early pregnancy that can be distinguished from common nausea and vomiting of pregnancy by considerable ketonuria on urine dipstick, greater than $5 \%$ weight loss, and onset at less than 16 weeks' estimated gestational age. ${ }^{2}$

Although hyperemesis in pregnancy is typically $\dot{\omega}$ self-limiting, it can also be secondary to a multiple 9 gestation, molar pregnancy, cholecystitis, pyelonephritis, pancreatitis, hepatitis, and, rarely, hyperthyroidism. ${ }^{3}$ A focused history, physical examination, and laboratory evaluation can usually rule out abnormalities of the gallbladder, liver, kidney, and pancreas, and obstetric sonography can rule out multiple gestations and molar pregnancy.

Thyroid function studies can rule out hyperthyroidism, as they did in this case. In a normal pregnancy, thyroid-binding globulin levels are elevated above nonpregnant levels because of estrogenstimulated synthesis. As a result, total $T_{3}$ and $T_{4}$ levels are elevated, but biochemically active free $T_{3}$ and free $T_{4}$ levels are within normal limits. In contrast, pathologic hyperthyroidism (eg, secondary to Graves disease) is marked by elevated free $T_{3}$ and free $\mathrm{T}_{4}$ and suppressed TSH levels. The same pattern of thyroid function abnormalities occurs in THHG (Table 1).

Because it is impossible to distinguish pathologic hyperthyroidism from THHG by thyroid function tests alone, the diagnosis of THHG rests on four criteria:

1. Abnormal thyroid function tests developing in the context of byperemesis gravidarum

By definition, THHG develops in the context of hyperemesis gravidarum. Thyroid function abnormalities that precede the development of hyperemesis gravidaurm or persist after its resolution should trigger a search for alternative diagnoses. 


\section{No evidence of prepregnancy bypertbyroidism}

The possibility that abnormal thyroid function tests could indicate previously undiagnosed hyperthyroidism can be ruled out by a thorough prepregnancy review of systems. Graves disease, the most common cause of pathologic hyperthyroidism in women of childbearing age, only rarely develops in pregnancy; most patients with Graves disease will have symptoms that predate their pregnancy. ${ }^{4} \mathrm{~Pa}$ tients with THHG, in contrast, will have no prepregnancy symptoms. ${ }^{5}$ This review of symptoms of hyperthyroidism should include nervousness, sweating, heat intolerance, hyperactivity, tremor, weakness, diarrhea, tachycardia, and increased appetite. $^{6}$

A history of hyperemesis in previous pregnancies, however, as in this case, is often found in patients with THHG. ${ }^{5}$

\section{Absence of physical examination findings consistent with bypertbyroidism}

Whether because of the brief or mild nature of the hyperthyroidism of THHG, the classic physical examination findings associated with hyperthyroidism are not seen. ${ }^{2,7}$ These include goiter, lid lag, onycholysis, proximal muscle weakness, and the exophthalmos of Graves disease. Patients with THHG might, however, have a mild resting tachycardia and mild tremor. ${ }^{5}$

\section{Negative thyroid autoantibody titers}

Graves disease can be ruled out by evaluation of thyroid autoantibody titers. Antithyroid peroxidase and anti-TSH receptor antibodies are often positive in Graves disease but negative in THHG (Table 1$).^{5}$

\section{Incidence}

There are no published reports on the incidence of THHG. An estimate can be extrapolated from data on hyperemesis gravidarum and from the relatively small case series reports on THHG. Hyperemesis gravidarum occurs in about 1 in 2000 pregnancies. ${ }^{8}$ The largest published case series of THHG found evidence of hyperthyroidism consistent with THHG in approximately $70 \%$ of cases of hyperemesis gravidarum. ${ }^{2}$ Based on these data, THHG might occur in up to 0.7 of 2000 pregnancies.

Separate data put the incidence of thyrotoxicosis in pregnancy at 1 in $2000 .^{9}$ It is unclear whether THHG is included in this figure. If not, then THHG could account for $40 \%$ of hyperthyroidism in pregnancy. If included, THHG accounts for $70 \%$ of cases of hyperthyroidism in pregnancy.

\section{Mecbanism}

Since the association between hyperemesis gravidarum and biochemical markers of hyperthyroidism was first described in $1982,{ }^{10}$ investigation into the mechanism of THHG has focused on the role of human chorionic gonadotropin. Chorionic gonadotropin is known to act directly on TSH receptors to stimulate excess hormone production. Through a separate pathway, it could also act to increase estradiol levels that in turn trigger vomiting. ${ }^{11}$ Further studies have focused on an asialo subtype of chorionic gonadotropin that appears to have an increased affinity for TSH receptors. ${ }^{12}$ Additionally, a mutation in the TSH receptor conferring hypersensitivity to chorionic gonadotropin could play a role in some cases of $\mathrm{THHG}^{13}$ As chorionic gonadotropin levels in pregnancy naturally decline in the second trimester, both hyperemesis and thyroid function abnormalities of THHG resolve.

\section{Natural History and Treatment}

THHG is a self-limiting condition. Thyroid function tests typically return to normal by 18 weeks' estimated gestational age, although emesis can persist beyond 18 weeks. ${ }^{2}$ No treatment is required other than supportive care for the symptoms and consequences (eg, volume depletion) associated with hyperemesis gravidarum. There is no evidence of adverse outcomes in pregnancies complicated by THHG, and there is no evidence that treatment with antithyroid medications (eg, propylthiouracil) is warranted, although its use has been described in isolated case reports. Thyroid function tests can be repeated until they return to normal.

\section{Conclusion}

THHG is a benign condition masquerading as pathologic hyperthyroidism. Diagnosis is based on (1) abnormal thyroid function tests developing in the context of hyperemesis gravidarum, (2) no evidence of prepregnancy hyperthyroidism, (3) absence of physical examination findings consistent with hyperthyroidism, and (4) negative thyroid autoantibody titers (antithyroid peroxidase and TSHreceptor antibodies). Diagnosis and management 
are well within the scope of care provided by the family physician.

\section{References}

1. Crump WJ, Aten LA. Hyperemesis, hyperthyroidism, or both? J Fam Pract 1992;35:450, 453-6.

2. Goodwin TM, Montoro M, Mestman JH. Transient hyperthyroidism and hyperemesis gravidarum: clinical aspects. Am J Obstet Gynecol 1992;167:648 -52.

3. Krentz AJ, Redman H, Taylor KG. Hyperthyroidism associated with hyperemesis gravidarum. $\mathrm{Br} \mathrm{J}$ Clin Pract 1994;48(2):75-6.

4. Goodwin TM, Hershman JM. Hyperthyroidism due to inappropriate production of human chorionic gonadotropin. Clin Obstet Gynecol 1997;40:32-44.

5. Goodwin TM, Mestman J. Transient hyperthyroidism of hyperemesis gravidarum. Cont Ob/Gyn 1996; June:65-78.

6. Klein I, Trzepacz PT, Roberts M, Levey GS. Symptom rating scale for assessing hyperthyroidism Arch Intern Med 1988;148:387-90.

7. Thyroid disease in pregnancy. ACOG Technical
Bulletin. Number 181 - June 1993. Int J Gynaeco Obstet 1993;43:82-8.

8. Mazzaferri EL. Evaluation and management of com mon thyroid disorders in women. Am J Obstet Gy necol 1997;176:507-14.

9. Mestman JH. Hyperthyroidism in pregnancy. Clino Obstet Gynecol 1997;40:45-64.

10. Bouillon R, Naesens M, Van Assche FA, et al. Thy roid function in patients with hyperemesis gravida rum. Am J Obstet Gynecol 1982;143:922-6.

11. Goodwin TM, Montoro M, Mestman JH, Pekary AE, Hershman JM. The role of chorionic gonadotropin in transient hyperthyroidism of hyperemesisw gravidarum. J Clin Endocrinol Metab 1992;75: 1333-7.

12. Yoshimura M, Hershman JM. Thyrotropic action of human chorionic gonadotropin. Thyroid 1995;5 425-34.

13. Rodien $P$, Bremont C, Sanson ML, et al. Familia $\underset{\omega}{\omega}$ gestational hyperthyroidism caused by a mutant thy rotropin receptor hypersensitive to human chorionic gonadotropin. N Engl J Med 1998;339:1823-6. 